\title{
Blue rubber bleb nevus syndrome
}

\author{
Julia N. Mayba BSc MD, Kyle Cullingham MSc MD
}

- Cite as: CMAJ 2019 July 29;191:E841. doi: 10.1503/cmaj.190173

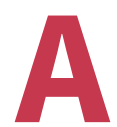

31-year-old man presented to the dermatology clinic with a lifetime history of painful blue nodules that were distributed throughout his body and bled easily. His uncle had similar lesions, as did his late father. The lesions had been biopsied previously; however, no conclusive diagnosis was made. The patient did not have a history of epistaxis, hematemesis, hematuria or blood in his stool but did have iron deficiency anemia. On physical examination, he had about 20 blue nodules distributed among the upper and lower extremities, abdomen and buttocks (Figures $1 \mathrm{~A}$ and $1 \mathrm{~B}$ ). Clinically, his presentation was suspicious for blue rubber bleb nevus syndrome.

We biopsied a nodule from his left forearm; histopathologic examination confirmed a benign cavernous vascular lesion, compatible with blue rubber bleb nevus. We referred the patient to the general surgery department for a discussion about gastrointestinal screening and considered starting treatment with sirolimus.

Blue rubber bleb nevus syndrome is an uncommon disease with fewer than 200 reported cases and is characterized by multiple venous malformations on the skin and visceral organs. ${ }^{1}$ It may be inherited autosomal dominantly, with mutations in TEK, the gene encoding tyrosine-protein kinase receptor 2 (TIE2), but may also occur sporadically. ${ }^{1,2}$ The differential diagnosis for the syndrome includes hereditary hemorrhagic telangiectasia, Klippel-Trenaunay syndrome and Maffucci syndrome. ${ }^{1}$

Patients with blue rubber bleb nevus syndrome may present with fatigue from hematemesis, melena or rectal bleeding. The gastrointestinal lesions may rupture spontaneously, causing acute hemorrhage and, potentially, death. However, most gastrointestinal bleeding is slow, chronic and occult. ${ }^{3}$

For minor or intermittent gastrointestinal bleeding, conservative treatment with blood transfusions and iron supplementation is suggested. ${ }^{1}$ For clinically significant hemorrhage, surgical resection, endoscopic sclerosis, laser photocoagulation and treatment with interferon have been suggested. ${ }^{1}$ Other medical treatments include corticosteroids, propranalol and sirolimus. Specifically, sirolimus has shown rapid reduction in the size of gastrointestinal lesions and cessation of massive gastrointestinal bleeding. ${ }^{1}$

At present, there are no recommendations for gastrointestinal screening for blue rubber bleb nevus syndrome; however, screening may be prudent in patients with iron deficiency anemia, such as in our patient.

\section{References}

1. Jin X-L, Wang Z-H, Xiao X-B, et al. Blue rubber bleb nevus syndrome: a case report and literature review. World J Gastroenterol 2014;20:17254-9.

2. Soblet J, Kangas J, Nätynki M, et al. Blue rubber bleb nevus (BRBN) syndrome is caused by somatic TEK (TIE2) mutations. J Invest Dermatol 2017;137:207-16.

3. Dobru D, Seuchea N, Dorin M, et al. Blue rubber bleb nevus syndrome: case report and literature review. Rom J Gastroenterol 2004;13:237-40.

\section{Competing interests: None declared.}

This article has been peer reviewed.

The authors have obtained patient consent.

Affiliation: Department of Medicine, University of Saskatchewan, Saskatoon, Sask.

Correspondence to: Julia Mayba, juliamayba@shaw.ca 